Meta

Journal des traducteurs

Translators' Journal

\title{
Acculturation et dénomination des langues africaines
}

\section{Nazam Halaoui}

Volume 54, numéro 3, septembre 2009

URI : https://id.erudit.org/iderudit/038315ar

DOI : https://doi.org/10.7202/038315ar

Aller au sommaire du numéro

Éditeur(s)

Les Presses de l'Université de Montréal

ISSN

0026-0452 (imprimé)

1492-1421 (numérique)

Découvrir la revue

Citer cet article

Halaoui, N. (2009). Acculturation et dénomination des langues africaines. Meta, 54(3), 566-574. https://doi.org/10.7202/038315ar

\section{Résumé de l'article}

Nombreuses sont les langues africaines qui, à l'heure actuelle, connaissent deux dénominations, l'une donnée par le colonisateur européen, l'autre, plus récemment, par les langagiers. Tandis que la première paraît étrangère en raison du processus de transcription, la seconde se veut authentique et fait appel aux ressources de la langue elle-même. L'étude présente cet état de fait pour trois langues d'Afrique de l'Ouest et montre que ces dénominations ne tiennent pas compte du nom de la langue en usage dans la langue elle-même, qu'elles sont des abus de langage et qu'elles résultent, en fait, d'une imposition, à la langue, de la culture du colonisateur ou de celle des langagiers.
Ce document est protégé par la loi sur le droit d'auteur. L'utilisation des services d’Érudit (y compris la reproduction) est assujettie à sa politique d'utilisation que vous pouvez consulter en ligne.

https://apropos.erudit.org/fr/usagers/politique-dutilisation/ 


\title{
Acculturation et dénomination des langues africaines
}

\author{
NAZAM HALAOUI \\ Université de Montréal, Montréal, Canada \\ nazam.halaoui@umontreal.ca
}

\begin{abstract}
RÉSUMÉ
Nombreuses sont les langues africaines qui, à l'heure actuelle, connaissent deux dénominations, l'une donnée par le colonisateur européen, l'autre, plus récemment, par les langagiers. Tandis que la première paraît étrangère en raison du processus de transcription, la seconde se veut authentique et fait appel aux ressources de la langue elle-même. L'étude présente cet état de fait pour trois langues d'Afrique de l'Ouest et montre que ces dénominations ne tiennent pas compte du nom de la langue en usage dans la langue elle-même, qu'elles sont des abus de langage et qu'elles résultent, en fait, d'une imposition, à la langue, de la culture du colonisateur ou de celle des langagiers.
\end{abstract}

\begin{abstract}
At the present time, several African languages have two names, the first one being given by the european colonizer, the other one, more recently, by language professionals and researchers. The first denomination looks foreign due to the transcription process, the second is intended to be authentic. This study analyses such a situation for three West African languages and shows that the names used in the languages themselves have been ignored. In fact, the denominations of both types have been imposed by the culture of the colonizer and by that of the linguists.
\end{abstract}

\section{MOTS-CLÉS/KEYWORDS}

dénomination de langue, acculturation, colonisation, glossonyme, Afrique de l'Ouest language denomination, acculturation, colonization, glottonym, West Africa

\section{Introduction}

Nombreuses sont, en Afrique noire francophone, les réalités qui bénéficient de deux dénominations. Dans le cas général, l'une est en usage dans la langue de la communauté et du lieu considérés et elle y justifie d'une existence plutôt ancienne: de ce fait, on est en droit d'estimer qu'elle a été attribuée à la réalité en question par les Africains eux-mêmes. L'autre est une transcription française de la première, plus ou moins conforme à la phonétique et à la phonologie de la langue africaine en question. Elle n'est pas reconnue par celle-ci et elle apparaît aux locuteurs africains comme étrangère, du moins à ceux qui ne sont pas totalement dominés par l'acculturation et qui gardent en eux une certaine africanité: on estime alors que cette dénomination a été retenue par le colonisateur français au moment de la conquête, sinon pendant l'ère coloniale. Il en est ainsi de nombreux patronymes (par exemple Jàra, Diarra...), de 
nombreux toponymes (Bafilaben, Bafoulabe...), ainsi que de nombreux glossonymes, dont certains constituent l'objet de la présente étude.

En effet, en Afrique de l'Ouest, trois langues mandées (Platiel 1978; Houis 1981a; Dwyer 1989)1 ${ }^{1}$ parmi les plus répandues possèdent, chacune, deux dénominations. Celles-ci constituent un cas particulier de la question soulevée: en effet, si l'une de ces dénominations a été effectivement retenue par le colonisateur au moment de la conquête, l'autre a été proposée et adoptée récemment par les langagiers (linguistes, terminologues, alphabétiseurs, etc.). On a ainsi, au Mali (Soudan sous la colonisation), bambara et bamanan, au Burkina Faso (autrefois Haute-Volta) et en Côte d'Ivoire, dioula et jùla, et, en Guinée, malinké et màninka ${ }^{2}$. Ces dénominations ont ceci de particulier qu'elles ne correspondent nullement aux noms que ces langues se donnent à elles-mêmes: ils existent pourtant, mais ils ont été tout simplement ignorés, et par les Français, et, ce qui est beaucoup plus grave, par les Africains euxmêmes. Étant donné que ces langues possèdent un nom africain couramment utilisé par leurs propres locuteurs, l'objet de ces quelques pages est de présenter les faits tels qu'ils sont observables aujourd'hui, puis de montrer que ces dénominations constituent des abus de langage et qu'elles résultent d'une imposition de la culture de certains individus à la langue.

\section{Les mots sources}

Les mots sources sont des mots appartenant à l'une ou l'autre des langues mandées à l'étude ${ }^{3}$, dans lesquelles ils justifient chacun d'une existence ancienne. Il s'agit de bamanan, de jùla et de màninka. Ils sont à l'origine des dénominations qui sont présentées et analysées plus loin, car ils leur ont donné naissance et ils en constituent la raison d'être.

En raison de son schème tonal, le premier de ces mots connaît deux réalisations, [bamanã] et [bamànã]. L'une ou l'autre peut apparaître quand le mot constitue la base simple d'un nom. Par contre, seule la première apparaît quand le mot entre dans la formation de la base composée ou dérivée d'un nom. On retiendra le mot dans sa première réalisation, laquelle renverra indifféremment aux deux réalisations. Ce mot appartient au lexique de la langue dominante du Mali (Ouane 1986; Dumestre 1995; Maiga 2000). On n'entrera pas dans les considérations d'ordre étymologique, qui n'ont d'ailleurs jamais été acceptées, et qui voudraient que, dans sa seconde réalisation, ce mot soit un composé: par conséquent, on admettra qu'il constitue une base lexicale simple, assimilable à un lexème, qui montre une aptitude à s'associer à un morphème nominant et, donc, à donner un nom. Il s'agit donc d'une base nominale. En tant que base simple, ce mot désigne l'individu qui appartient à l'ethnie parlant la langue elle-même, à laquelle le mot appartient. Le lexème bamanan peut s'associer à un autre lexème et donner une base composée. Celle-ci peut être une base complétive, bamanan-dugu, /village, pays de bamanan/, ou bamanan-bugu, /case de bamanan/, ou encore bamanan-fanga, /pouvoir de bamanan/. Elle peut aussi être une base spécificative, donc appositive, bamanan-muso, /bamanan femme/, ou qualificative, bamanan-fin, /bamanan noir/. Le lexème bamanan peut aussi s'associer à un morphème dérivatif et donner une base dérivée. On a pour exemples, bamanan-na, /bamananmilieu/, ou bamanan-ya, /bamanan-qualité/. On voit, qu'au sein d'une base composée ou d'une base dérivée, le sens du lexème bamanan s'élargit, sans pour autant cependant que ne soit oblitérée la désignation de l'individu. 
Le mot jùla appartient, quant à lui, à la langue mandée du sud-ouest du Burkina Faso, dominante du point de vue du nombre de ses locuteurs (Nikiéma et Vinou 1978; Prignitz 1996). Il s'agit de l'une des principales langues véhiculaires du pays, et Bobo-Dioulasso est la principale agglomération où elle est parlée. Le même mot appartient aussi à une langue mandée parlée sous plusieurs formes en Côte d'Ivoire, en tant que langue véhiculaire dans tout le pays, ou langue de terroir dans la moitié nord de celui-ci (Lafage 1982; Kouadio 1993; Tera 1996). On peut reconnaître au moins deux référents au mot jùla. En effet, celui-ci réfère à un individu en tant qu'individu appartenant à l'ethnie qui parle la langue. Il réfère aussi à un individu dont l'activité de subsistance est le commerce, il désigne alors souvent le colporteur. Ce trait du mot jùla a permis de faire sur sa provenance l'hypothèse selon laquelle il aurait été à l'origine un mot arabe signifiant colporteur : il serait donc un emprunt à cette langue. Par ailleurs, il s'agit aussi d'une base nominale simple, pouvant s'associer à d'autres formants pour donner des bases lexicales composées ou dérivées.

Enfin, le mot màninka appartient à la langue mandée dominante en Haute Guinée (Guinée 1986; Diallo 1993). La principale agglomération où cette langue est parlée est la ville de Kankan. Ce mot, ainsi adopté aujourd'hui, apparaît comme une base lexicale simple, assimilable à un lexème, donc indécomposable en unités du même niveau. En fait, il résulte de la contraction des deux formants en lesquels s'organisait auparavant une base lexicale dérivée, à savoir le lexème mànden ou màndin, lequel désigne un espace géographique, situé approximativement au sud-ouest du Mali et au nord-est de la Guinée, et le morphème de type dérivatif $-\underline{k a}$, dont la valeur exprime la provenance. De ce fait, la base lexicale dérivée référait à l'individu qui provient du Manden. Aujourd'hui, la base lexicale simple renvoie toujours au même type d'individu, sans pour autant, en raison de la contraction, être explicite sur la localisation géographique, comme l'indiquait la base lexicale dérivée. Sans entrer les détails, le mot màninka, tout comme on l'a montré pour le mot bamanan, est aujourd'hui une base nominale simple qui peut s'associer à d'autres formants et donner des bases composées ou des bases dérivées.

\section{Les dénominations des langues}

Les dénominations des langues sont les noms actuellement utilisés pour désigner les langues mandées en question. Elles sont de deux types, coloniales ou authentiques. Les premières sont dites coloniales (le qualifiant étant bien sûr utilisé sans péjoration aucune), car elles ont été attribuées aux langues par le colonisateur lors de la conquête ou pendant l'ère coloniale. Les secondes sont qualifiées d'authentiques d'abord parce qu'il s'agit de mots appartenant effectivement aux langues elles-mêmes, ensuite parce que le motif qui guidait les langagiers lors de leur adoption était la recherche d'une certaine authenticité africaine, le terme étant pris dans son sens scientifique et non idéologique.

Les dénominations coloniales sont bambara, dioula et malinké (Delafosse 1929, 1955, 1972; Houis 1981a, 1981b). Le mot bambara est la transcription effectuée par les Français selon les normes d'écriture de leur propre langue, du mot bamanan présenté plus haut, et peut-être celle du mot bambara (Delafosse 1904: 140), en usage alors dans des populations musulmanes de l'ouest du Mali pour désigner certaines ethnies de race noire, dont les Bamanan. Signalons aussi que, jusqu'aux années 50, à Bamako, 
on retrouvait encore le mot bambara, réalisé avec une voyelle [a] suivie de la consonne $[\mathrm{m}]$, donc sans aucune nasalisation de la voyelle, et un [r] roulé, dans la bouche des Peuls qui faisaient alors un usage lacunaire de la langue parlée par les Bamanan. Si aujourd'hui ceux-ci ont une maîtrise certaine de cette langue, il n'en était pas ainsi à cette époque. Or, on sait que nombreux étaient les interprètes d'origine peule qui étaient en service dans l'administration coloniale. Y a-t-il là une explication de l'origine du mot bambara, lequel aurait d'abord été une mauvaise réalisation de bamanan dans la bouche de locuteurs non natifs? Nul ne le sait. Ceci étant dit, dans les écrits en langue française, ce mot réfère à la fois à l'individu (désigné par le mot bamanan dans sa propre langue) et à la langue parlée par ce dernier. Il est utilisé en tant que base nominale, un Bambara, les Bambara, le bambara, etc. Il participe aussi à la formation de syntagmes, notamment qualificatifs et en fonction de qualifiant, comme dans langue bambara, femme bambara, pays bambara, etc. Il en est de même des deux autres dénominations coloniales, dioula (qui a aussi été noté dyoula) et malinké. Elles sont aussi des transcriptions françaises, les deux premières, du mot jùla, la dernière, de l'un des mots màninka, présenté plus haut, ou màli-nke. Ce dernier mot est, par hypothèse au moins, formé de màli, qui désigne un espace géographique, et de - $\underline{n k e}$, qui serait, tout comme $-\underline{k a}$, un morphème dérivatif qui exprime une valeur de provenance, et qui est encore aujourd'hui attesté et productif en soninké, langue mandée principalement parlée dans le sud-est de la Mauritanie, le nord-est du Sénégal et l'ouest du Mali. Tout comme le mot bambara, dans les travaux en français, ces dénominations coloniales réfèrent chacune à l'individu et à la langue, et elles apparaissent dans des syntagmes, notamment qualificatifs, et avec une fonction de qualifiant.

Les dénominations authentiques, quant à elles, sont les mots bamanan, jùla et màninka. Ces mots sont identiques, sur le plan formel et en partie sur le plan sémantique, aux mots sources. Ils sont utilisés essentiellement par les langagiers depuis quelques décennies et dans les travaux en langue française, avec les mêmes sens et les mêmes référents que, respectivement, les mots coloniaux bambara, dioula et malinké. Selon une estimation de l'auteur (qui demande à être vérifiée, car on ne dispose d'aucune étude sur la question), le mot màninka serait apparu en Guinée au début des années 60, avec l'aménagement linguistique de ce pays qui œuvrait à l'introduction des langues nationales dans l'enseignement. Le mot bamanan a vu le jour au Mali au début des années 70 avec l'utilisation des langues nationales dans l'alphabétisation. Enfin, le mot jùla s'est imposé, essentiellement sous la plume des linguistes, au Burkina Faso et en Côte d'Ivoire dans la deuxième moitié des années 70, sans pour autant que l'usage de dioula ne disparaisse dans ce dernier pays. Ces mots sont utilisés comme base nominale. Le Bamanan et le bamanan, ou le Jùla et le jùla, ou encore le Màninka et le màninka réfèrent, respectivement, à l'individu et à la langue. Ils apparaissent aussi dans des syntagmes qualificatifs, et la langue bamanan réfère à la langue parlée par le Bamanan, la langue jùla à celle parlée par le Jùla, et la langue màninka à celle qui l'est par le Màninka.

\section{La marque de la culture}

Étant donné l'existence des mots sources, bamanan, jùla et màninka , et les modalités de leur utilisation dans les langues auxquelles ils appartiennent, l'analyse des mots coloniaux (bambara, dioula et malinké) et des mots authentiques (bamanan, jùla et 
màninka) aboutit nécessairement à deux conclusions: ces mots sont à considérer comme des abus de langage et, fait essentiel, les processus de dénomination à la source des mêmes mots sont d'origine culturelle.

On a vu que chacun des mots coloniaux détenait au sein des écrits en français trois propriétés référentielles différentes, celles de désigner l'individu, de référer à la langue parlée et d'assumer la fonction de qualifiant. Si ces mots tiennent leur première propriété des mots dont ils proviennent, lesquels désignent effectivement l'individu, on ne saurait dire de quoi ils tiennent les deux autres, car, on l'a vu plus haut, ni bamanan, ni jùla, ni màninka ne désignent la langue ni n'assument la fonction de qualifiant. Ces mots ont été transcrits et on a assigné aux transcriptions des usages que n'ont pas les mots d'origine. On peut donc considérer qu'il y a là abus de langage. Cependant, on peut aussi trouver une excuse aux auteurs francophones en observant que, ces mots étant entrés dans la langue française, ils peuvent alors, à l'instar d'autres mots du français de la même catégorie, désigner la langue et assumer la fonction de qualifiant.

Concernant maintenant les dénominations authentiques, on a aussi vu que ceuxci détenaient les trois propriétés détenues par les noms coloniaux. Si ces mots sont analysés comme effectivement authentiques, car appartenant chacun à l'une des langues mandées en question, ils sont donc les mots sources qui ont été présentés plus haut. Tel étant le cas, on ne peut leur accorder qu'une seule de ces propriétés, la première, car étant la seule à être attestée dans ces langues. Les deux autres ne le sont pas. Si, maintenant, les mêmes mots sont admis avec les trois propriétés, alors on ne peut leur reconnaître une authenticité quelconque et on ne peut qu'admettre qu'il s'agit de mauvaises traductions des mots coloniaux, de calques. On a en fait refusé les mots coloniaux, mais on a pris le sens général de chacun de ceux-ci et on a proposé des mots authentiques pour rendre ce sens. Bambara se dit effectivement bamanan, dioula se dit jùla et malinké se dit màninka. Ce faisant, les langagiers qui sont à l'origine de ces dénominations n'ont pas tenu compte des usages, donc des propriétés, mais aussi des contraintes auxquelles ces mots, sous leur forme authentique, sont soumis dans les langues auxquelles ils appartiennent. Par conséquent, ils ont outrepassé les droits que leur accordaient ces langues et ont imposé à ces mots des usages qu'ils n'avaient pas. Il y a ici aussi, à n'en pas douter, abus de langage.

Il n'est guère possible d'exiger une écriture phonétique des mots coloniaux (l'alphabet phonétique international n'ayant été établi qu'en 1888, quelques années seulement avant l'entrée des Français dans les pays concernés), mais on peut cependant faire remarquer que ces mots sont écrits selon les règles de l'orthographe du français. À preuve, le $m$ qui succède et nasalise le $a$, mais précède le $b$ dans bambara, la suite di pour rendre [j] qui, à l'initiale des mots français, ne semble être attesté que dans quelques emprunts, la suite ou pour transcrire [u], etc. D'autre part, quand les colonisateurs ont adopté ces dénominations, ils se sont situés dans l'esprit de la langue française et ne semblent pas s'être souciés de savoir si la langue était désignée par un nom autre que celui qui désignait l'individu. Sachant que sur les territoires conquis vivaient, selon le lieu, les Bamanan, les Jùla ou les Màninka, ils se sont contentés de ces noms, les ont transcrits tant bien que mal, et ont utilisé ces transcriptions pour désigner les langues. Ce faisant, ils n'ont fait qu'appliquer à celles-ci la pratique connue du français, à savoir l'utilisation du même mot pour dénommer l'individu et sa langue. Ainsi, tout l'individu originaire de France (un Français) et la langue qui 
est parlée dans ce pays (le français) sont désignés par une seule et même base lexicale, seules une majuscule et une minuscule initiales créant la différence. On a donc retenu une seule base lexicale pour désigner tant l'une ou l'autre de ces langues mandées que l'individu les parlant, alors que seul ce dernier pouvait être dénommé par cette base lexicale. Le mot d'origine a certes été respecté, mais, à travers la transcription et les usages qui lui ont été assignés, s'est effectuée la francisation du mot, son acculturation doit-on dire. Il y a là une imposition de la culture française aux dénominations des langues mandées ici concernées.

Par ailleurs, violant l'orthographe phonologique des mots d'origine et détenant des propriétés inconnues des langues d'origine, ces dénominations coloniales sont à considérer aujourd'hui comme des emprunts, largement intégrés, du français à ces langues. De ce fait, on ne saurait continuer à voir en eux des mots appartenant à l'une ou l'autre de ces langues mandées. En fait, ils ne sont pas et ils n’ont jamais été des mots de ces langues. Ces mots sont à considérer comme des mots français, et donc comme des mots étrangers à ces mêmes langues. Telle est la situation qui a incité les langagiers à proposer des mots authentiques. En effet, quand on traite de telle ou telle autre langue, pourquoi utiliser une appellation de cette langue qui lui étrangère, de surcroît attribuée à elle par des colonisateurs, quand cette langue dispose d'une appellation qui lui est propre? La connaissance des langues mandées et l'expertise des linguistes mandés allant grandissant, les langagiers ont réagi à l'utilisation des dénominations coloniales de leurs langues. Ils ont souhaité écarter celles-ci au profit de mots appartenant à ces langues, c'est-à-dire de dénominations authentiques. Ils ont de ce fait imposé leur culture aux langues, ce qui, au demeurant, paraissait légitime, l'intention étant d'associer une culture mandée à une langue mandée.

Cependant, ce qui n'est pas légitime, c'est que, ce faisant, ils n'ont pas du tout associé une culture mandée à une langue mandée, ils ont imposé aux langues mandées leur propre culture de lettrés francophones, leur culture d'acculturés doit-on dire, acquise à travers la colonisation, l'éducation et surtout l'apprentissage et l'intériorisation de la langue française. C'est ainsi qu'en adoptant les dénominations authentiques, on a créé inconsciemment des barbarismes, du type le bamanan ou la langue bamanan, le jùla ou la langue jùla, ou encore le maninka ou la langue maninka, pour désigner ces langues mandées, conformément à une pratique connue en français et intériorisée chez les langagiers acculturés, mais véritablement inconnue dans ces langues. Jamais les mots bamanan, jùla ou màninka n’ont désigné une langue quelconque, jamais ces mots ne sont utilisés à titre de qualifiant. Ces mots sont certes authentiques du point de vue de leur forme, mais dans le processus d'adoption dont ils ont été l'objet, ils ont baigné dans la culture des langagiers, laquelle leur a assigné des sens et des référents qu'ils n'ont pas en tant que mots authentiques. Il y a ici aussi une imposition de la culture des langagiers aux dénominations des langues, imposition résultant d'une acculturation patente.

\section{Conclusion}

Si la critique est nécessaire pour le développement de la connaissance, elle ne saurait contribuer à la réalisation d'un tel objectif sans la formulation de propositions concrètes. Comme conclusion de ces quelques pages, on ne peut que préconiser l'adoption de l'une des deux positions suivantes: 
- Soit on estime qu'on parle et qu'on écrit le français, et on est alors en droit de suivre pleinement la pratique coloniale. Celle-ci devient légitime dans un tel cadre, et les mots bambara, dioula et malinké doivent être considérés comme des mots français, avec tous les usages que permet la langue française. Dans ce cas, chacun de ces mots désignera l'individu, réfèrera à la langue et assumera la fonction de qualifiant. Ces mots seront aussi soumis à la morphologie de la langue française et chacun d'eux pourra connaître un singulier, mais aussi un pluriel noté selon l'usage par la suffixation de la lettre $s$, un masculin, mais aussi un féminin noté selon l'usage par la suffixation de la lettre $e$, etc.

- Soit on convient qu'on doit être scientifique et authentique, et on souhaite alors africaniser les dénominations. Dans ce cas, on doit se soumettre à la langue elle-même, ce qui signifie qu’on ne doit admettre les dénominations bamanan, jùla et màninka que pour désigner l'individu, qu'on doit bannir l'usage de ces dénominations pour désigner la langue ou assumer une fonction de qualifiant, enfin et surtout qu'on doit se résoudre à utiliser les dénominations en usage dans la langue elle-même pour désigner la langue, à savoir bamanankan, jùlakan et màninkakan. Telles sont en effet les dénominations authentiques des langues, les dénominations réelles doit-on dire.

TABleau 1

Propriétés des mots et des dénominations

\begin{tabular}{|c|c|c|c|}
\hline & $\begin{array}{l}\text { Désignation de } \\
\text { l'individu }\end{array}$ & $\begin{array}{c}\text { Désignation de la } \\
\text { langue }\end{array}$ & $\begin{array}{l}\text { Fonction de quali- } \\
\text { fiant }\end{array}$ \\
\hline $\begin{array}{l}\text { Sources } \\
\text { - bamanan } \\
\text { - jùla } \\
\text { - màninka }\end{array}$ & $\begin{array}{l}\text { oui } \\
\text { oui } \\
\text { oui }\end{array}$ & $\begin{array}{l}\text { non } \\
\text { non } \\
\text { non }\end{array}$ & $\begin{array}{l}\text { non } \\
\text { non } \\
\text { non }\end{array}$ \\
\hline $\begin{array}{l}\text { Coloniales } \\
\text { - bambara } \\
\text { - dioula } \\
\text { - malinké }\end{array}$ & $\begin{array}{l}\text { oui } \\
\text { oui } \\
\text { oui }\end{array}$ & $\begin{array}{l}\text { oui } \\
\text { oui } \\
\text { oui }\end{array}$ & $\begin{array}{l}\text { oui } \\
\text { oui } \\
\text { oui }\end{array}$ \\
\hline $\begin{array}{l}\text { Authentiques } \\
\text { - bamanan } \\
\text { - jùla } \\
\text { - màninka }\end{array}$ & $\begin{array}{l}\text { oui } \\
\text { oui } \\
\text { oui }\end{array}$ & $\begin{array}{l}\text { oui } \\
\text { oui } \\
\text { oui }\end{array}$ & $\begin{array}{l}\text { oui } \\
\text { oui } \\
\text { oui }\end{array}$ \\
\hline $\begin{array}{l}\text { Proposées 1: } \\
\text { dénominations } \\
\text { coloniales } \\
\end{array}$ & $\begin{array}{l}\text { considérées comme } \\
\text { emprunts intégrés au } \\
\text { français }\end{array}$ & $\begin{array}{l}\text { comme mots français, } \\
\text { avec toutes les } \\
\text { implications }\end{array}$ & $\begin{array}{l}\text { morphologiques, } \\
\text { syntaxiques et } \\
\text { orthographiques }\end{array}$ \\
\hline $\begin{array}{l}\text { Proposées 2: } \\
\text { dénominations réelles } \\
\text { - bamanan } \\
\text { - bamanankan } \\
\text { - jùla } \\
\text { - jùlakan } \\
\text { - màninka } \\
\text { - màninkakan }\end{array}$ & $\begin{array}{l}\text { oui } \\
\text { non } \\
\text { oui } \\
\text { non } \\
\text { oui } \\
\text { non }\end{array}$ & $\begin{array}{c}\text { non } \\
\text { oui } \\
\text { non } \\
\text { oui } \\
\text { non } \\
\text { oui }\end{array}$ & $\begin{array}{l}\text { non } \\
\text { non } \\
\text { non } \\
\text { non } \\
\text { non } \\
\text { non }\end{array}$ \\
\hline
\end{tabular}

La question traitée dans la présente étude n'est spécifique aux ni aux dénominations des langues mandées, ni aux glossonymes en général. L'onomastique africaine, ce qui nous ramène à la situation générale introduite plus haut, est affectée par les différences observables entre les noms sources et véritablement authentiques et les dénominations coloniales. Il n'y a pas à blâmer le colonisateur, car les langues africaines ne bénéficiant à cette époque ni d'alphabet ni d'orthographe, celui-ci a utilisé de manière légitime l'alphabet et l'orthographe dont il disposait, celui et celle de la langue française. Ceci étant, maintenant que ces langues disposent de tels instruments 
et sont écrites, et sans pour autant impliquer la suppression pure et simple des noms coloniaux, lesquels meublent les écrits depuis plus d'un siècle, un retour à une certaine authenticité serait souhaitable, ne serait-ce que pour la science et la connaissance, car l'écriture actuelle oblitère une large information relative à la signification des noms. Sans prendre le risque de se tromper, on peut affirmer que l'orthographe actuelle interdit l'accès à la connaissance des réalités désignées, alors que l'association à celle-ci d'une orthographe conforme à la langue faciliterait largement cet accès. Une telle démarche demande cependant une certaine rigueur, dont le fondement réside dans une soumission totale du langagier à la langue, laquelle requiert principalement d'être exempte de toute acculturation.

\section{NOTES}

1. Les titres indiqués en référence visent à illustrer l'usage des mots et donner la situation des langues, plutôt qu'à étayer le sujet, lequel, à notre connaissance, n’a aucunement été analysé.

2. Les langues en question font usage de deux tons, marqués sur la première voyelle du mot. L'accent grave symbolise le ton bas, l'absence d'accent le ton haut.

3. Le présent texte étant écrit en français, en raison de la position qui apparaîtra dans la conclusion, les dénominations coloniales seront utilisées. Cependant, pour les besoins de l'argumentation, on s'efforcera, quand cela semblera possible, d'éviter ces dénominations, au risque de faire souffrir le style.

\section{RÉFÉRENCES}

Bailleul, Charles (1996): Dictionnaire bambara-français. Bamako: Editions Donniya.

Coulibaly, Bakary (1984): Le jùla véhiculaire de Haute-Volta: phonologie, morphologie, syntaxe et règles de transcription orthographique. Paris: Université René Descartes.

Delafosse, Maurice (1901): Essai de manuel pratique de la langue mandé ou mandingue. Paris: Leroux.

Delafosse, Maurice (1904): Vocabulaires comparatifs de plus de 60 langues ou dialectes parlées à la Côte d'Ivoire et dans les régions limitrophes. Paris: Leroux.

Delafosse, Maurice (1929): La langue mandingue et ses dialectes, tome I. Paris: Geuthner.

Delafosse, Maurice (1955): La langue mandingue et ses dialectes, tome II. Paris: Geuthner.

Delafosse, Maurice (1972): Haut-Sénégal-Niger. Paris: Maisonneuve et Larose.

Diallo, Alpha Mamadou (1993): Le français en Guinée: une situation en plein changement. In: Didier de Robillard et Michel Beniamino, dir. Le français dans l'espace francophone. Paris: Champion, 229-242.

Dumestre, Gérard (1995): La dynamique des langues au Mali: le trinôme langues régionalesbambara-français. In: Gérard Dumestre et Cécile CANut, dir. Stratégies communicatives au Mali: langues régionales, bambara, français. Paris: Didier Erudition.

Dwyer, David (1989): Mande. In: John Bendor-Samuel et Rhonda L. Hartell, dir. The Niger-Congo languages: a classification and description of Africa's largest language family. Lanham: University Press of America, 47-65.

Grégoire, Claire (1986): Le maninka de Kankan. Éléments de description phonologique. Paris: Agence de coopération culturelle et technique - Musée royal de l'Afrique centrale.

Guinée (1986): Guinée. In: Guy Dumas, dir. Promotion et intégration des langues nationales dans les systèmes éducatifs. Bilan et inventaire. Conférence des ministres de l'éducation des pays d'expression française (CONFEMEN). Paris: Champion, 185-197.

HouIs, Maurice (1971): Anthropologie linguistique de l'Afrique noire. Paris: PUF.

Hours, Maurice (1981a): Les langues du groupe mande. In: Jean Perrot, dir. Les langues dans le monde ancien et moderne, Afrique subsaharienne, pidgins et créoles, texte. Paris: CNRS, 67-73.

Houis, Maurice (1981b) : Le bambara. In: Jean Perrot, dir. Les langues dans le monde ancien et moderne, Afrique subsaharienne, pidgins et créoles, texte. Paris: CNRS, 75-82. 
Kouadio, N'Guessan Jérémie (1993): Côte d'Ivoire. La situation linguistique. Diagonales. 26:42-44.

LAfAge, Suzanne (1982): Esquisse des relations interlinguistiques en Côte d'Ivoire. Bulletin de l'Observatoire du français contemporain en Afrique noire. 3:9-27.

Maiga, Hamidou (2000): La situation sociolinguistique du Mali. In: Pierre Dumont et Christine SAntodomingo, dir. La coexistence des langues dans l'espace francophone, approche sociolinguistique. Universités francophones, Actualité scientifique. Montréal: AUPELFUREF, 159-163.

Ministère de l'Éducation Nationale et de la Culture du Burkina Faso (1995): Lexique de base jula. Ouagadougou: INA.

Nikiema, Norbert et Ye, Vinou (1978): La Haute-Volta. In: Daniel Barreteau, dir. Inventaire des études linguistiques sur les pays d'Afrique noire d'expression française et sur Madagascar. Paris: CILF, 435-442.

OuAne, Adama (1978): Quelques données sociolinguistiques sur le Mali. In: Daniel BarreteAu, dir. Inventaire des études linguistiques sur les pays d'Afrique noire d'expression française et sur Madagascar. Paris: CILF, 375-387.

Ouane, Adama (1986): Aperçu sociolinguistique du Mali. In: Guy Dumas, dir. Promotion et intégration des langues nationales dans les systèmes éducatifs. Bilan et inventaire. Conférence des ministres de l'éducation des pays d'expression française (CONFEMEN). Paris: Champion, 531-538.

Platiel, Suzanne (1978): Les langues mandé. In: Daniel Barreteau, dir. Inventaire des études linguistiques sur les pays d'Afrique noire d'expression française et sur Madagascar. Paris: CILF, 41-62.

Prignitz, Gisèle (1996): Contrastes et paradoxes du Burkina Faso, pays essentiellement multilingue et résolument francophone, In: Didier de Robillard et Michel Beniamino, dir. Le français dans l'espace francophone. Paris: Champion, 547-575.

Tera, Kalilou (1996): Le dioula véhiculaire de Côte d'Ivoire. Expansion et développement. Cahiers ivoiriens de recherche linguistique. 20:5-33. 